\title{
DISTRIBUTION OF PFCRT HAPLOTYPES AND IN-VIVO EFFICACY OF CHLOROQUINE IN TREATMENT OF UNCOMPLICATED P. FALCIPARUM MALARIA BEFORE DEPLOYMENT OF ARTEMISININ COMBINATION THERAPIES IN URBAN POPULATION OF KOLKATA, INDIA
}

\author{
PABITRA SAHA ${ }^{1}$, SHRABANEE MULLICK ${ }^{1}$, SUBHASISH K. GUHA ${ }^{1}$, SONALI DAS ${ }^{1}$, \\ SWAGATA GANGULY ${ }^{1}$, MITALI CHATTERJEE ${ }^{5}$, ASIT BISWAS², DILIP K. BERA ${ }^{1}$, \\ ABHIRAM CHAKRAVORTY ${ }^{1}$, BASUDEB MUKHERJEE ${ }^{4}$, MADHUSUDAN DAS ${ }^{3}$, \\ PRATIP K. KUNDU1, KRISHNANGSHU RAY ${ }^{1}$ AND ARDHENDU K. MAJI** \\ ${ }^{1}$ Calcutta School of Tropical Medicine, 108, C. R. Avenue, Kolkata 700073 , India \\ 2Department of Health \& Family Welfare, Government of West Bengal, Swasthya Bhawan, Salt Lake City, Kolkata-700092, \\ India \\ 3Department of Zoology, University of Calcutta, 45, Ballygunge Circular Road, Kolkata-700 019, India \\ ${ }^{4}$ Kolkata Municipal Corporation, 5, S. N. Banerjee Road, Kolkata - 700013 \\ 5Department of Microbiology, N.R.S. Medical College, Kolkata \\ *Corresponding author. E-mail: ardhendu_maji@yahoo.com, Telephone: +91 9433630077, Fax: +91 3322414915
}

Received: September 09, 2011; Accepted: October 19, 2011

\begin{abstract}
A total of 101 P. falciparum positive patients were enrolled from urban population of Kolkata, India to determine the therapeutic efficacy of chloroquine as per 28 days follow-up schedule of WHO, 2003. All parasite strains were analyzed for $P$. falciparum chloroquine resistance transporter (pfcrt) haplotypes using DNA sequencing methodology. The PCR corrected chloroquine resistant $P$. falciparum was very high $(76.3 \%, 95 \% \mathrm{Cl} 0.642-0.832)$ of which early treatment failure was $(10 \%)$, and late treatment failure was $(66.3 \%)$. K76T mutation was found in all parasite strains irrespective of therapeutic outcomes. Both the Venezuelan (SVMNT) and Southeast Asian (CVIET) haplotypes were prevalent in the study population with predominance of South East Asian haplotype (87.1\%). The present study showed that incidence of CQ resistant $P$. falciparum malaria in Kolkata was very high and well above the WHO recommended cut-off level for change of drug policy. Recently introduced Artimisinine Combination Therapy by the Government of India to treat all $P$. falciparum cases is an appropriate step.
\end{abstract}

Key Words: Malaria, Plasmodium falciparum, Chloroquine resistant, Pfcrt, Haplotype

\section{Introduction}

During the second half of $20^{\text {th }}$ century chloroquine $(C Q)$ became the antimalarial agent of choice due to its efficacy and low toxicity [1]. Then the resistance to $C Q$ was developed in Plasmodium falciparum and subsequently spread over almost all the endemic areas of the world. $\mathrm{CQ}$ resistance in $P$. falciparum has contributed to the global resurgence of malaria and its consequent morbidity and mortality. Resistance of $P$. falciparum to $C Q$ was first reported from South-East Asia in 1957 and then in South America in 1959 [2]. From India, CQ-resistant $P$. falciparum was first reported in 1973 from Diphu area of Karbi Anglong district of Assam [3]. Several reports of resistance were subsequently confirmed from the different parts of India [4-8]. Due to increased CQ-resistance in 2002 National Anti Malaria Programme (NAMP) introduced SulfadoxinePyrimethamine (SP) as the second line option to treat falciparum malaria cases. But SP resistance has developed quickly in other parts of the world following its wide spread use $[9,10]$ and also in different parts of India
$[11,12]$. As the CQ-resistance has reached significantly high levels, National Vector Born Disease Control Programme (NVBDCP) has recently introduced Artemisinin Combination Therapy (ACT) for the treatment of uncomplicated falciparum malaria throughout India.

$P$. falciparum chloroquine resistance transporter ( $p f c r t)$ gene is associated with the CQR phenotype in $P$. falciparum which is located on chromosome 7 [13]. The pfcrt gene encodes an integral membrane protein located in parasite digestive vacuole membrane [13]. During haemoglobin digestion haem molecules are released in the digestive vacuole and detoxified by the formation of haemozoin and $C Q$ is suggested to interfere with this process $[14,15]$. $P$. falciparum $C Q R$ is suggested to involve mechanisms whereby $\mathrm{pH}$ sensitive physiologic processes inhibit formation of toxic $C Q$ haematin complexes in favor of haemozoin [15] or $\mathrm{CQ}$ efflux reduces drug concentration to the levels that are no longer parasiticidal [16-18]. 
In different parts of world 15 genetic polymorphisms have been identified in the pfcrt gene, which are linked to CQR in P. falciparum [19-21]. Mutations of particular interest are in the region of pfort gene encompassing codons 72-76. Among these codons the amino acid substitution at pfcrt codon $76(\mathrm{~K} \rightarrow \mathrm{T})$ has been shown to have the strongest association with the CQR phenotype [22]. Substitutions in the wild type allele, encoding CVMNK, give rise to several resistant variants, of which the most common are CVIET in South-East Asia \& Africa and SVMNT, which has been reported in South America [13] and Asia [23], but rarely in Africa [24].

In the present study, DNA sequencing technique was employed to study the distribution of different $C Q$ resistant genotypes at positions codons 72 - 76 of pfort gene in the study population and evaluate the correlation of in-vivo chloroquine resistance with the pfort K76T mutation. Samples were collected during a clinical study in 2009, before the deployment of ACT in India.

\section{Materials and Methods \\ Study Areas}

The study was conducted in the Kolkata district of West Bengal state in India, which is situated along the banks of the river Hoogly and lies at $22.30^{\circ} \mathrm{N}$ latitude and 88.30 $\mathrm{E}$ longitude and at an altitude of $17 \mathrm{ft}$ above the sea. The study was carried out in two different malaria clinics - one at the Malaria Clinic of Calcutta School of Tropical Medicine (Central Kolkata) and the other at Malaria Clinic of Kolkata Municipal Corporation, Ward No. 81 (South Kolkata). The study was carried out during January, 2009 - March, 2009. The malaria transmission intensity was seasonal (July-December) with the predominance of falciparum malaria and the annual parasite index in 2008 was 10.69 .

\section{Screening of Patients and Recruitment}

The febrile patients from surrounding localities attending the Malaria Clinic of Calcutta School of Tropical Medicine and the Malaria Clinic of Kolkata Municipal Corporation were screened for malarial parasite by examining Giemsa stained thick and thin peripheral blood smear (PBS). During the study period, a total of 1812 patients with fever were screened, of them 454 tested positive for malaria (200 P. falciparum, $250 P$. vivax, and four mixed infections of both $P$. falciparum and $P$. vivax). The patients with confirmed $P$. falciparum mono-infection confirmed by pan-Plasmodium antigen lactate dehydrogenase ( $\mathrm{pLDH}$ ) test, were explained about the study protocol and requested to participate into the study. Those who fulfilled the following inclusion criteria (Age: over 6 months; $P$. falciparum mono-infection with asexual parasite count - 1000-100 000/ul; axillary temperature $\geq 37.5^{\circ} \mathrm{C}$ or history of fever during past 24 $\mathrm{h}$; ability to swallow oral medication; absence of signs of severe falciparum malaria; absence of febrile condition due to diseases other than malaria; absence of regular medication, which might interfere with antimalarial pharmacokinetics; a negative pregnancy test or not breastfeeding) as per WHO protocol (2009) [25] were enrolled following obtaining written, informed consent. The medical history including presenting symptoms, current medications and previous antimalarial use were recorded. A complete physical examination was performed and case record form was completed for each patient. Clinical history, examination and other investigational data were recorded. Blood was collected for parasitology and molecular biology studies, using standard protocol.

\section{Treatment and Follow up}

A standard three-day dose of chloroquine $(25 \mathrm{mg} / \mathrm{kg} \mathrm{bw}$, NVDCP supply) according to body weight with biscuits and glass of water was provided by the study team under observation. Patients were observed for 1 hour after treatment. In case of vomiting within 30 minutes of administration, a full dose was repeated and beyond that period up to one hour, half a dose was repeated. Single dose of primaquine (PQ) $0.75 \mathrm{mg} / \mathrm{Kg}$ of body weight were given on day 1.

After enrollment all patients were given a follow-up schedule for attending the clinic on days 1, 2, 3, 7, 14, 21 and 28 after treatment. Patients were advised to return on any day during follow-up period if symptoms recurred and not to wait for schedule visit day. During the followup visits the axillary temperature was recorded. In addition, thorough clinical examinations were carried out to identify the presence of any signs of severe and complicated malaria. On the event of occurrence of any such complications patients were withdrawn from the study. The treatment failure cases were treated by AS+SP combination as per Indian National Drug Policy for Malaria. In this study, no signs of severe and complicated malaria were recorded in the study patients. Blood films were drawn for parasite count on Day 1, 2, 3, $7,14,21, \& 28$ or any other day if the patient spontaneously returned to the clinic. For genetic study the blood samples were collected in EDTA coated vial on day 0 and on any other day with reappearance of parasitaemia.

\section{Study end points}

The in vivo study outcomes were classified according to the WHO 28-day protocol [25]. The clinical endpoints were: (1) early treatment failure (ETF), (2) late treatment failure (LTF), which included late clinical failure (LCF) and late parasitological failure (LPF) and (3) adequate clinical and parasitological response (ACPR).

\section{Ethical approval}

The Ethics Committee of the Calcutta School of Tropical Medicine, Kolkata, approved the study protocol. Informed consent of the patients or their legal guardians was obtained before recruitment of the patients in the study.

\section{Laboratory Method \\ Microscopic blood examination and parasite count}

Parasite counts were done on Giemsa-stained thick films and the number of parasites per 200 WBCs was counted. Assuming a WBC count to be $8,000 / \mu \mathrm{l}$ of blood, 
parasitaemia was calculated and expressed as per $\mu$ of blood. A thick smear was diagnosed as negative on initial review if no parasites were seen in 100 oil immersion fields and $10 \%$ of positive and negative slides were cross-checked.

\section{DNA extraction and PCR Genotyping}

Genomic DNA of $P$. falciparum was isolated from $200 \mu \mathrm{l}$ EDTA blood that were collected on day 0 and on any other day on reappearance of parasitaemia, using QiaAmp DNA mini kit (Qiagen, Hilden, Germany) as per manufacturer's instructions.

To differentiate between recrudescence and re-infection of the paired samples of treatment failure cases, genotyping was performed with a nested PCR assay based on the amplification of msp1 and msp2and glurp as described in detail elsewhere [26, 27].

In the primary reaction, the used oligonucleotide primers spanned the entire genetic segments i.e., block 2 for $m s p 1$ and block 3 for $m s p 2$ and R II block of glurp. In the nested reaction, separate primer pairs targeted the respective allelic types of msp1 (K1, MAD20, and RO33) and msp2 (FC27 and 3D7).

PCR amplifications were performed on a Thermal Cycler (Perkin Elmer, Branchburg, NJ, USA). All amplification reactions were carried out in a final volume of $20 \mu \mathrm{l}$ which included $2 \mu$ of DNA template (genomic DNA for the primary reactions and the product of the primary reaction for the secondary amplification). Oligonucleotide primers were used at final concentrations of $0.3 \mu \mathrm{M}$ in both primary as well as secondary reactions. The reaction mixture contained PCR Buffer, $0.2 \mathrm{mM}$ concentration of each of the four deoxynucleoside triphosphates, and $0.75 \mathrm{U}$ of AmpliTaq polymerase (Perkin Elmer, Branchburg, NJ, USA).

The reactions were carried out in the presence of $2 \mathrm{mM}$ $\mathrm{MgCl}_{2}$ for all oligonucleotide combinations except the msp 2 nested PCR, for which a concentration of $1.5 \mathrm{mM}$ $\mathrm{MgCl}_{2}$ was used. The cycling parameters for the PCR were as follows; an initial denaturation at $94^{\circ} \mathrm{C}$ for 2 minutes followed by 30 cycles of -denaturation at $94^{\circ} \mathrm{C}$ for 30 seconds, annealing at $54^{\circ} \mathrm{C}$ for primary PCR $\left(50^{\circ}\right.$ $\mathrm{C}$ for $m s p 2$ nested and $59^{\circ} \mathrm{C}$ for $m s p 1$ and glurp nested) for 1 minute, extension at $72^{\circ} \mathrm{C}$ for 2 minutes. The final extension was done at $72^{\circ} \mathrm{C}$ for 5 minutes. The PCR products were stored at $4^{\circ} \mathrm{C}$ until further analysis. Nested PCR products were analyzed by electrophoresis using $2 \%$ agarose gels (performed in TBE buffer).

All the distinguishable allelic variants for each marker paired samples were loaded side by side. The gels were stained with Ethidium Bromide and visualized under UV illumination and documented by Gel-Doc system. Gel photographs were analyzed by visual comparison of DNA fragments on base line and recurrent samples.

A recrudescent infection was defined as one that showed a match in size of at least one allele for both the msp1, msp2 and glurp genes on day 0 and on the day of recurrent parasitaemia.
PCR amplification and sequencing of pfcrt gene

$P$. falciparum chloroquine resistance transporter ( $p f c r t)$ is $3.1 \mathrm{~kb}$ gene with 13 exons, of which exon 2 contains the codons 72-76. Exon 2 of pfort gene was amplified by PCR using the oligonucleotide primer pair PFCRT-P1 5'GGCTCACGTTTAGGTGGA-3' and PFCRT-P2 5'TGAATTTCCCTTTTTATTTCCAAA-3' [28], which gives a 264-basepair product corresponding to amino acid residues 32 to 119. PCR amplifications were carried out in a final volume of $35 \mu$ which include $3 \mu$ of genomic DNA as template. The reaction mixture contained PCR buffer, $0.2 \mathrm{mM}$ of dNTPs, $2.5 \mathrm{mM} \mathrm{MgCl} 2,0.3 \mu \mathrm{M}$ of each of the primer and $1.5 \mathrm{U}$ of Ampli Taq polymerase (Perkin Elmer, Branchburg, NJ, USA). The cycling parameters for the PCR was an initial denaturation at $94^{\circ} \mathrm{C}$ for 3 minutes followed by 45 cycles of denaturation $94^{\circ} \mathrm{C}$ for 30 seconds, annealing $58^{\circ} \mathrm{C}$ for 30 seconds and extension $60^{\circ} \mathrm{C}$ for 1 minute. The final extension was done at $60^{\circ} \mathrm{C}$ for 3 minutes.

The quality and concentration of PCR products for each sample were ascertained by agarose gel electrophoresis. The PCR products were purified from the agarose gel by use of the QIAEX-II Gel Extraction Kit (Qiagen, Hilden, Germany), in accordance with the manufacturer's instructions. The sequencing was done using the BigDye terminator 3.1 (Applied Biosystems) with forward primer (PFCRT-P1).

\section{Sequence analysis}

The sequences were analyzed using the software Bioedit Sequence Alignment Editor version 7.0.5.2. The sequences were then aligned using the online multiple sequence alignment tool ClustalW (available at: http: //www.ebi.ac.uk/clustalw).

\section{Statistical analysis}

The data was entered into a standard data entry programme designed by Global Malaria Programme and analyzed by Kaplan-Meier survival curve according to WHO standard procedures (http://www.who.int/malaria/resistance). The 95\% confidence interval was calculated by Dimension Research calculator (http://www.dimensionresearch.com/resources/ resources overview.html).

\section{Results}

\section{Study population}

During the study period, we screened 1812 patients presenting with fever, among them 454 tested positive for malaria (200 P. falciparum, $250 P$. vivax, and four mixed infections of both $P$. falciparum and $P$. vivax). Of the 200 P. falciparum, 101 patients met the enrolment criteria for the study. Their baseline characteristics are described in Table 1.

In vivo drug efficacy

Originally 101 patients were enrolled from the two different malaria clinics of the study area (50 from Malaria Clinic of Calcutta School of Tropical Medicine and 51 from the Malaria Clinic of Kolkata Municipal Corporation, Ward no 81). Among the 101 patients, eight 
patients withdrew consent from the study and seven patients were lost to follow-up, leaving 86 in our per protocol analysis. Eighty-six (85.1\%) patients completed the $\mathrm{CQ}$ treatment course and complete the 28 day follow-up schedule and reached one of the study endpoints (Figure 1). Among the patients who completed the follow-up, nineteen $(22.1 \%)$ exhibited adequate clinical and parasitological response (ACPR). The remaining patients failed to treatment and these were classified using standard WHO definitions. The PCR uncorrected incidence of $\mathrm{CQ}$ failure for uncomplicated $P$. falciparum was 67 (77.9\%); among them ETF, LCF and LPF were 8 (9.3\%), $48(55.8 \%)$ and $11(12.8 \%)$ respectively (Table 2).

Out of fifty nine apparent therapeutic failures (LTF) cases, four samples could not be evaluated due to inability to amplify the parasitic DNA for $m s p 1, m s p 2$ and glurp genotyping. Two LTF cases were reclassified as 're-infection' on the basis of DNA genotyping and remaining fifty three recurrent infections were classified as 'recrudescence'. The PCR corrected cumulative incidence of $\mathrm{CQ}$ failure for uncomplicated $P$. falciparum was therefore $76.3 \%(61 / 80,95 \% \mathrm{Cl} 64.2-83.2)$ and the therapeutic efficacy was $23.7 \%(19 / 80,95 \%$ Cl 16.8 - 35.8) (Table 2).

\section{Mutation analysis of the pfort gene}

DNA was isolated from blood samples, collected on day 0, of all 101 enrolled patients and were subjected to PCR amplification and sequencing of pfort gene. Sequencing was done successfully in 93 samples. In the present study, mutations of pfcrt gene were observed at positions 72 (87.1\%), 74 (12.9\%), 75 (12.9\%), 76 (100.0\%), and no mutations were observed at position 73 (Figure 2). The wild type, C72V73M74N75K76, was not detected in any of the samples studied. All the samples (including both $C Q$ sensitive and $C Q$ resistant) belonged to the mutant genotype. There were two mutant pfort haplotypes in the parasite population, S72V73M74N75T76 was highly prevalent (81/93; 87.1\%, 95\% Cl 80.29 - 93.91); C72V73I74E75T76 was present in $12.9 \%(12 / 93,95 \% \mathrm{Cl} 6.09$ - 19.71) patients. Interestingly, the mutant genotype S72V73M74N75T76 was prevalent in both $C Q$ sensitive $(13 / 18 ; 72.2 \%)$ and CQ resistant $(58 / 64 ; 90.6 \%)$ (Table 3). Further, the mutant genotype C72V73I74E75T76 was observed in $27.8 \%(5 / 18)$ of $\mathrm{CQ}$ sensitive isolates and $9.3 \%(6 / 64)$ among $C Q$ resistant isolates. These observations suggest that the mutant genotype S72V73M74N75T76 of the pfort gene is widely distributed among the $P$. falciparum isolates in the study area. However, the S72V73M74N75T76 genotype was more prevalent in ETF $(8 / 8 ; 100.0 \%)$ than LCF $(42 / 46 ; 94.1 \%)$, and LPF (8/10; 80.0\%). The C72V73I74E75T76 genotype was more prevalent in ACPR $(5 / 18 ; 27.8 \%)$ than LPF $(2 / 10$; $20.0 \%$ ), LCF (4/46; 8.6\%), and ETF (Table 3).

\section{Discussion}

Early and effective treatment of malaria is the cornerstone of malaria control, and appropriate selection of first- and second-line antimalarial medicines for country programmes is based entirely on the efficacy of the drugs against the malaria parasite. Monitoring the therapeutic efficacy of antimalarial drugs is, therefore, a fundamental component of treatment strategies. As the parasite evolves continuously to develop resistance against antimalarials, continuous global monitoring and reporting of drug efficacy and parasite resistance are needed [29].

In vivo studies remain the gold standard for monitoring antimalarial drug efficacy and are the primary source of information for the policy makers to formulate recommendations for malaria chemotherapy and prophylaxis. However, molecular surveillance can give an advanced indication that a particular drug may lose its efficacy in the near future. The present study was designed to determine the therapeutic efficacy of $C Q$ in $P$. falciparum malaria in Kolkata and distribution of pfcrt haplotypes in the study area.

A very high proportion of $P$. falciparum cases $(76.3 \%$, $95 \% \mathrm{Cl} 0.642-0.832$ ) were found to be resistant to Chloroquine and importantly about $10 \%$ of the total study cases were categorized as early treatment failure (ETF). In India, chloroquine was used as first line agent to treat all categories of malaria, till 2010. The long term use of the drug, often indiscriminately, is the most important factor for development of parasitic resistance. Similarly, a high prevalence of chloroquine resistant $P$. falciparum malaria was reported from different parts of the country [4-8]. Very recently, Mullick et al. [30] reported 61.2\% chloroquine resistant $P$. falciparum malaria from Jalpaiguri district of the same state where the current study was undertaken. So, the change of drug policy by the National Vector Born Disease Control Programme (NVBDCP) of India, from Chloroquine to ACT for all $P$. falciparum malaria cases was appropriate.

$P$. falciparum chloroquine resistance transporter ( $p f c r t)$ gene has been found to be associated with the CQR phenotype in $P$. falciparum. Research revealed that substitution of threonine $(T)$ for lysine $(K)$ at poison 76 (K76T) is the hallmark of CQR [31]. In the present study, mutant K76T was found in all parasite strains irrespective of Chloroquine response. Similarly a widespread high prevalence of the K76T mutation has also been reported elsewhere from India $[5,8,28,32]$ even in the $C Q$ responders. Several reports showed that the pfcrt mutation is necessary but not the only factor to predict the in-vivo response to $C Q$ [33]. Host immune status plays an important role in the clearance of $P$. falciparum infection, which may influence the in-vivo response to the drug [32]. Different haplotypes of pfort gene have been reported from different parts of the world. In the present study, two different haplotypes (amino acids 72 - 76) were detected. The Venezuelan haplotype SVMNT was predominant $(87 \%)$ over South East Asian haplotype CVIET (13\%) like in other studies from India [5,8].

Considering the WHO recommended cut-off level for change of drug policy and high incidence of $C Q$ resistant $P$. falciparum malaria in Kolkata, the present study is in 
agreement with the introduction of ACT by the Government of India in the therapeutic guideline. The mutant K76T was identified in all CQ resistant as well as sensitive strains. Further studies are indicated to elucidate the reason(s) for existence of K76T mutation among $C Q$ responders with respect to the host immunity, other mutations of pfort and pharmacokinetic characteristics of the drug.

Ethical approval: The study protocol was approved by the ethical committee of the Calcutta School of Tropical Medicine, Kolkata.

Conflicts of interest: The authors have no conflicts of interest concerning the work reported in this paper.

Authors' contributions: AKM, SKG and DKB conceptualized and designed the study protocol; PS, SM, SG , SD, PKK, BM and AB performed the clinical assessment and the in-vivo therapeutic efficacy study; $P$ $S$ and MD performed the PCR and sequencing analysis and interpretation of data; AKM, PS, SKG, PKK, AC, KR and DKB drafted the manuscript. All authors read and approved the final manuscript. AKM, SKG, and DKB are guarantors of the paper.

Acknowledgements: We are grateful to the Department of Health and Family Welfare, Government of West Bengal, India for funding the project. Special thanks to all patients who participated in this study. We are grateful to the Director, Calcutta School of Tropical Medicine, for his kind permission to publish the data.

\section{References}

[1] Cooper R.A., Hartwig C.L., and Ferdig M.T. (2005) Acta. Tropica., 94 (3), 170-180.

[2] Wernsdorfer W.H., and Kouznetsov R.L. (1980) Bull. World. Health. Organ., 58, 341-352.

[3] Sehgal P.N., Sharma M.I.D., Sharma S.L., and Gogoi S. (1973) J. Commu. Dis., 5, 175-180.

[4] Sathpathy S.K., Jena R.C., Sharma R.S., and Sharma R.C. (1997) J. Commu. Dis., 26, 145-151.

[5] Pati S.S., Mishra S., Mohanty S., Mahapatra D.N., Sahu P.K., Priyadarshi N., Kumar S., Sharma S.K., Tyagi P.K., Chitnis C.E., and Das B.S. (2007) Trans. R. Soc. Trop. Med. Hyg., 101, 650-654.

[6] Eapen A., Ravindran K.J., Joshi H., Dhiman R.C., Balavinayagam S., Mallick P.K., Kumar R., Rajendran C., Selvakumar A.D., and Dash A.P. (2007) Ann. Trop. Med. Parasitol., 101(4), 305-313.

[7] Srivastava H.C., Yadav R.S., Joshi H., Valecha N., Mallick P.K., Prajapati S.K., and Dash A.P. (2008). Ann. Trop. Med. Parasitol., 102(6), 471-480.

[8] Bharti P.K., Alam M.T., Boxer R., Shukla M.M., Gautam S.P., Sharma Y.D., and Singh N. (2010) Trop. Med. Int. Health., 15 (1), 33-40.

[9] Pinichpongse S., Doberstyn E.B., Cullen J.R., Yisunsri L., Thongsombun Y., and Thimasarn K. (1982) Bull. World. Health. Organ., 60, 907-912.
[10] Ejov M.N., Tun T., Aung S. and Sein, K. (1999) Bull. World. Health. Organ., 77, 244-249.

[11] Mohapatra P.K., Prakash A., Taison K., Negmu K., Gohain A.C., Namchoom N.S., Wange D., Bhattacharyya D.R., Goswami B.K., Borgohain B.K., and Mahanta J. (2005) Trop. Med. Int. Health., 10 (5), 478-483.

[12] Ahmed A., Lumb V., Das M.K., Dev V., Wajihullah., and Sharma Y.D. (2006) Antimicrob. Agents. Chemother., 50(11), 3934-3938.

[13] Fidock D.A., Nomura T., Talley A.K., Cooper R.A., Dzekunov S.M., Ferdig M.T., Ursos L.M.B., Sidhu A.B.S., Naudé B., Deitsch K.W., Su X.Z., Wootton J.C., Roepe P.D., and Wellems T.E. (2000) Mol. Cell., 6, 861-871.

[14] Goldberg D.E., Slater A.F., Cerami A., and Henderson G.B. (1990) Proc. Natl. Acad. Sci. USA., 87, 2931-2935.

[15] Dzekunov S.M., Ursos L.M., and Roepe P.D. (2000) Mol. Biochem. Parasitol., 110, 107-124.

[16] Saliba K.J., Folb P.I., and Smith P.J. (1998) Biochem. Pharmacol., 56, 313-320.

[17] Krogstad D.J., Gluzman I.Y., Kyle D.E., Oduola A.M., Martin S.K., Milhous W.K., and Schlesinger P.H. (1987) Science, 238, 1283-1285.

[18] Sanchez C.P., McLean J.E., Rohrbach P., Fidock D.A., Stein W.D., and Lanzer M. (2005) Biochemistry, 44, 9862-9870.

[19] Wellems T.E., Walker-Jonah A., and Panton L.J. (1991) Proc. Natl. Acad. Sci. USA., 88, 3382-3386.

[20] Su X., Kirkman L.A., Fujioka H., and Wellems T.E. (1997) Cell, 91, 593-603.

[21] Chen N., Kyle D.E., Pasay C., Fowler E.V., Baker J., Peters J.M., and Cheng Q. (2003) Antimicrob. Agents. Chemother., 47, 3500-3505.

[22] Mehlotra R.K., Fujioka H., Roepe P.D., Janneh O., Ursos L.M., Jacobs-Lorena V., McNamara D.T., Bockarie M.J., Kazura J.W., Kyle D.E., Fidock, D.A., and Zimmerman P.A. (2001) Proc. Natl. Acad. Sci. USA., 98, 12689-12694.

[23] Sutherland C.J., Haustein T., Gadalla N., Armstrong M., Doherty J.F., and Chiodini P.L. (2007) J. Antimicr. Chemother., 59, 1197-1199.

[24] Alifrangis M., Dalgaard M.B., Lusingu J.P., Vestergaard L.S., Staalsoe T., Jensen A.T., Enevold A., Rønn A.M., Khalil I.F., Warhurst D.C., Lemnge M.M., Theander T.G., and Bygbjerg I.C. (2006) J. Infect. Dis., 193, 1738-1741.

[25] World Health Organization, Geneva. (2003) Assessment and Monitoring of Antimalarial Drug Efficacy for the treatment of Uncomplicated Falciparum Malaria. World Health Organization, Geneva, WHO/HTM/ RBM/ 2003.50.

[26] Snounou G. (2002) Methods. Mol. Med., 72, 103116.

[27] Falk N., Maire N., Sama W., Owusu-Agyei S., Smith T., Beck H.P., and Felger I. (2006) Am. J. Trop. Med. Hyg., 74, 944-950.

[28] Vathsala P.G., Pramanik A., Dhanasekaran S., UshaDevi C., Pillai C.R., Subbarao S.K., Ghosh 
S.K., Tiwari S.N., Sathyanarayan T.S., Deshpande P.R., $\quad$ Mishra G.C., Ranjit M.R., Dash A.P., Rangarajan P.N., and Padmanabanet G. (2004) Am. J. Trop. Med. Hyg., 70(3), 256 -259.

[29] World Health Organization, Geneva, (2010) Global report on antimalarial drug efficacy and drug resistance: 2000-2010. World Health Organization, Geneva.

[30] Mullick S., Das S., Guha S.K., Bera D.K., Sengupta S., Roy D., Saha P., Biswas A., Das M., Ray K., Kundu P.K., and Maji A.K. (2011) Trop. Med. Int. Health., 16 (8), 929-935.
[31] Dittrich S., Alifrangis M., Stohrer J.M., Thongpaseuth V., Vanisaveth V., Phetsouvanh R., Phompida S., Khalil I.F., and Jelinek T. (2005) Trop. Med. Int. Health., 10, 1267-1270.

[32] Vinayak S., Biswas S., Dev V., Kumar A., Ansari M.A., and Sharma Y.D. (2003) Acta. Trop., 87, 287 293.

[33] Djimde A.A., Doumbo O.K., Traore O., Guindo A.B., Kayentao K., Diourte Y., Niare-Doumbo S., Coulibaly D., Kone A.K., Cissoko Y., Tekete M., Fofana B., Dicko A., Diallo D.A., Wellems T.E., Kwiatkowski D., and Plowe C.V., (2003) Am. J. Trop. Med. Hyg., 69, 558 - 563. 
Table 1- Base line characteristics of study patients

\begin{tabular}{ll}
\hline Characteristics & Study patients ( $\mathbf{n = 1 0 1 )}$ \\
\hline Sex: no. $(\%)$ & $58(57.4)$ \\
Male & $43(42.6)$ \\
Female & \\
& \\
Age category: no. (\%) & $29(28.7)$ \\
< 15 Year & $72(71.3)$ \\
>15 Year & \\
& \\
Age: year & 31.2 \\
Mean & $05-70$ \\
Range & \pm 13.4 \\
SD & $28.6-33.8$ \\
95\% Cl & \\
Temperature: ${ }^{0} \mathrm{C}$ & 37.7 \\
Mean & $37.5-38.2$ \\
Range & \pm 0.16 \\
SD & $37.66-37.73$ \\
95\% Cl & \\
Parasite count: no/ $\mu \mathrm{L}$ & 7700 \\
Mean & $1000-64000$ \\
Range & \pm 9191.4 \\
SD & $5907.5-9492.5$ \\
95\% Cl & \\
\hline
\end{tabular}

Table 2- Study outcomes CQ treated patients $(n=101)$

\begin{tabular}{lllll}
\hline \multirow{2}{*}{$\begin{array}{c}\text { Clinical } \\
\text { outcomes }\end{array}$} & \multicolumn{2}{c}{ PCR uncorrected } & \multicolumn{2}{c}{ PCR corrected } \\
\cline { 2 - 5 } ETF & \multicolumn{1}{c}{ No. of cases (\%) } & \multicolumn{1}{c}{$95 \% \mathrm{Cl}$} & \multicolumn{1}{c}{ No. of cases (\%) } & $95 \% \mathrm{Cl}$ \\
LCF & $8(9.3)$ & $4.1-17.5$ & $8(10.0)$ & $4.4-18.8$ \\
LPF & $48(55.8)$ & $44.7-66.5$ & $43(53.8)$ & $44.2-65.0$ \\
ACPR & $11(12.8)$ & $6.6-21.7$ & $10(12.5)$ & $6.2-21.8$ \\
Total Analysis & $19(22.1)$ & $13.9-32.3$ & $19(23.7)$ & $14.9-34.6$ \\
WTH & $\mathbf{8 6}$ & & 80 & \\
LFU & 8 & & 10 & \\
TOTAL & $7(14.9)$ & & $7(17.5)$ & \\
\hline
\end{tabular}

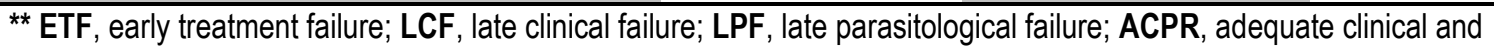
parasitological response; WTH, withdrawn; LFU, loss to follow-up.

Table 3- Prevalence pfcrt haplotypes among the CQ treated patients of study area

\begin{tabular}{|c|c|c|c|}
\hline \multirow{2}{*}{ Study Outcomes } & \multirow{2}{*}{$\begin{array}{l}\text { No. of isolates } \\
\text { analyzed for pfcrt }\end{array}$} & \multicolumn{2}{|c|}{ pfcrt haplotypes codon $72-76 \mathrm{n}(\%)[95 \% \mathrm{Cl}]$} \\
\hline & & $\mathbf{S}_{\text {AGt }} \mathbf{V}_{\text {GtA }} \mathbf{M}_{\text {AtG }} \mathbf{N}_{\text {AAT }} T_{\text {ACA }}$ & $\mathbf{C}_{\text {TGt }} \mathbf{V}_{\text {Gtal }}$ ItT $_{\text {GAA }} T_{\text {ACA }}$ \\
\hline $\begin{array}{c}\text { CQ Sensitive }(n=19) \\
\text { ACPR }(n=19)\end{array}$ & 18 & $13(72.2)$ & $5(27.8)$ \\
\hline $\begin{array}{l}\text { CQ Resistant }(n=67) \\
\text { LCF }(n=48) \\
\text { LPF }(n=11) \\
\text { ETF }(n=8)\end{array}$ & $\begin{array}{l}46 \\
10 \\
8\end{array}$ & $\begin{array}{l}42(91.4) \\
8(80.0) \\
8(100.0)\end{array}$ & $\begin{array}{l}4(8.6) \\
2(20.0) \\
-\end{array}$ \\
\hline $\begin{array}{l}\text { Unknown }(n=15) \\
\text { WTH+LFU }(n=15)\end{array}$ & 11 & $10(90.9)$ & $1(9.1)$ \\
\hline TOTAL (n=101) & 93 & $81(87.1)$ [80.29 - 93.91] & $12(12.9)[6.09-19.71]$ \\
\hline
\end{tabular}

** $\mathbf{n}$, number of isolates; ETF, early treatment failure; LCF, late clinical failure; LPF, late parasitological failure; ACPR, adequate clinical and parasitological response; WTH, withdrawn; LFU, loss to follow-up. 


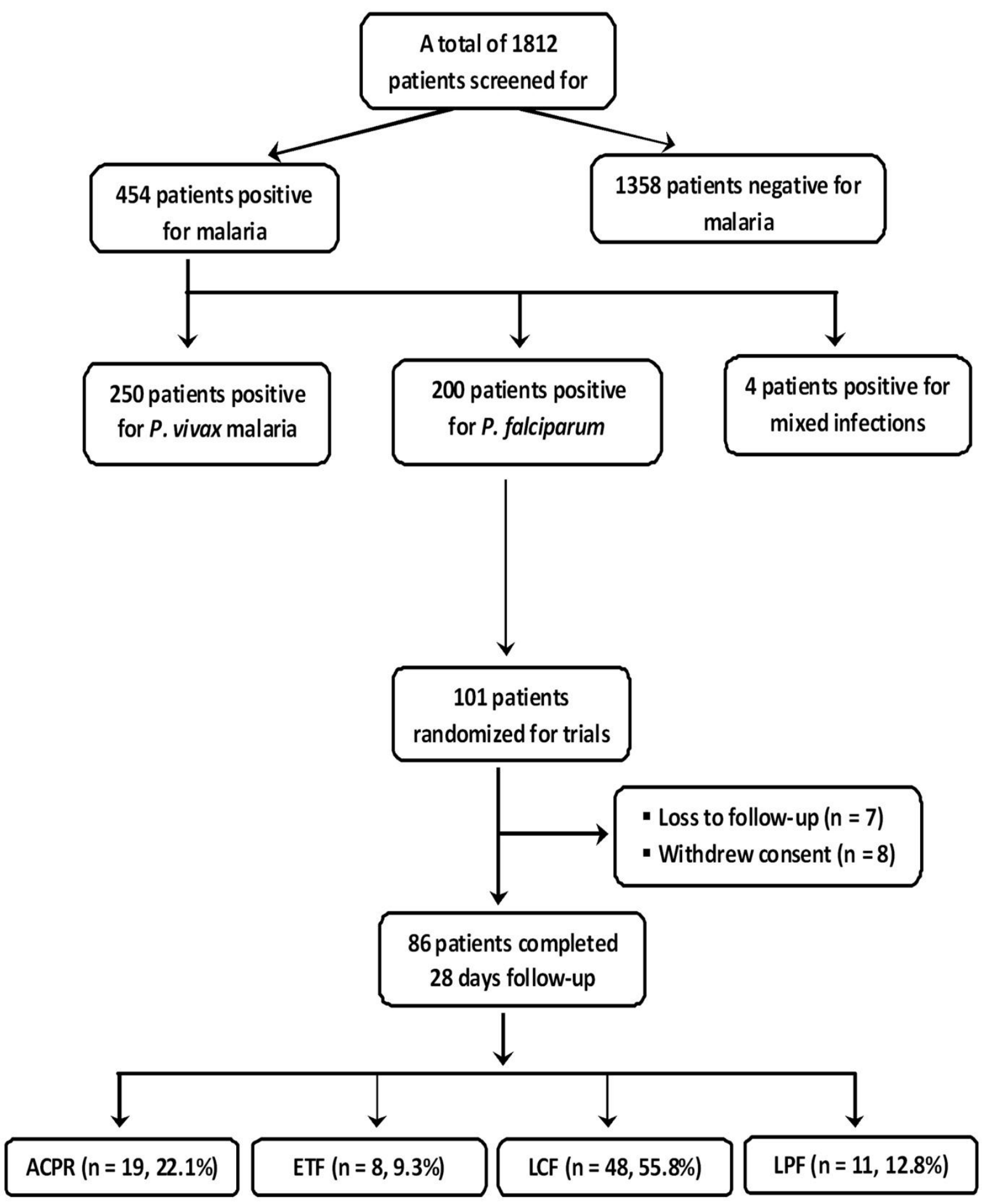

Fig. 1-Flow diagram of screening, follow-up and therapeutic outcomes of study patients 


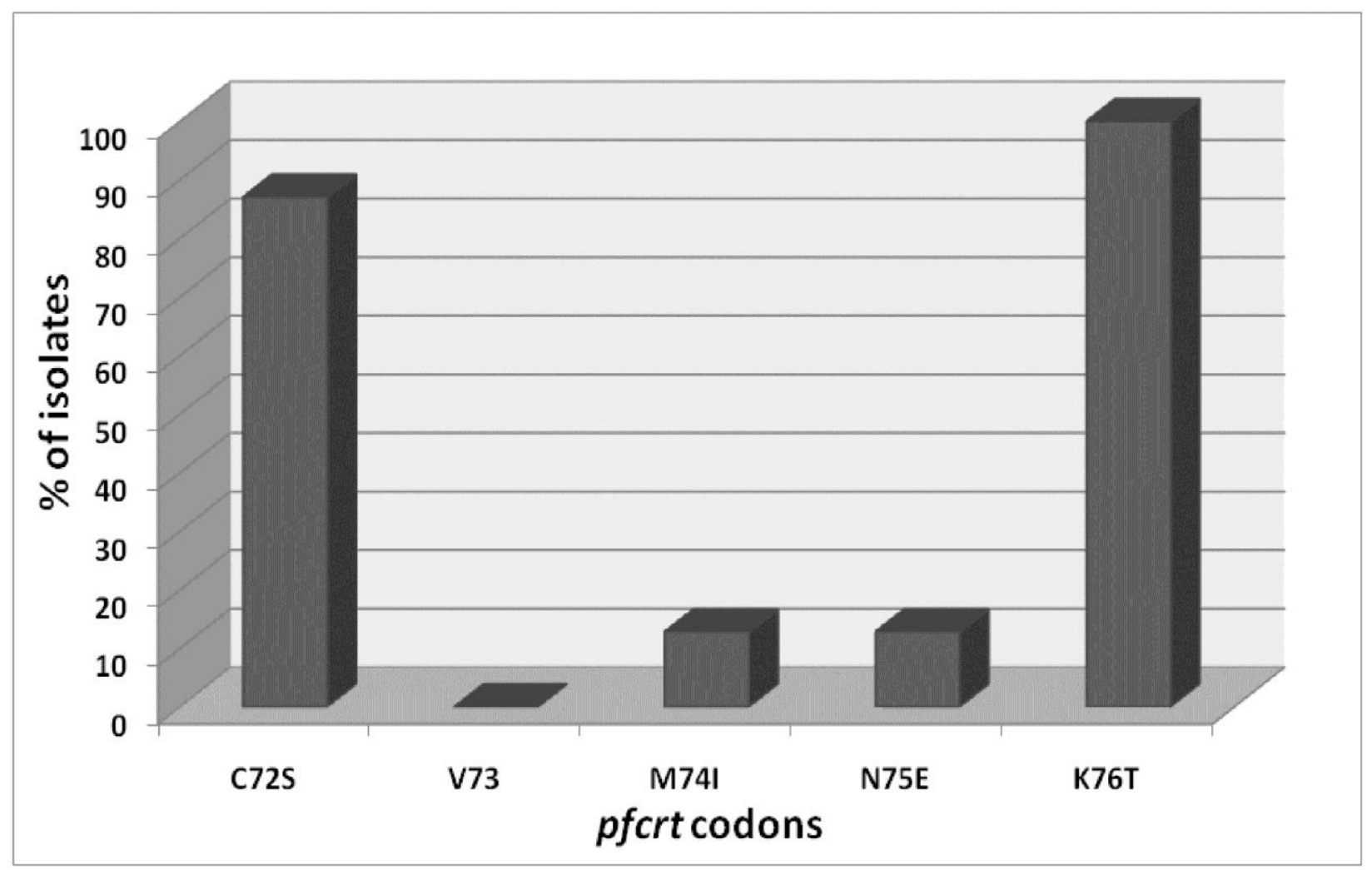

Fig. 2- Prevalence of pfcrt codons (72-76) among the $C Q$ treated patients $(n=101)$ of study area 